\title{
Total internal reflection fluorescence microscopy for determination of size of individual immobilized vesicles: Theory and experiment
}

\author{
Thomas Olsson, ${ }^{1}$ Vladimir P. Zhdanov, ${ }^{1,2, a)}$ and Fredrik Höök ${ }^{1, a)}$ \\ ${ }^{1}$ Section of Biological Physics, Department of Applied Physics, Chalmers University of Technology, \\ S-41296 Göteborg, Sweden \\ ${ }^{2}$ Boreskov Institute of Catalysis, Russian Academy of Sciences, Novosibirsk, Russia
}

(Received 15 March 2015; accepted 24 July 2015; published online 11 August 2015)

\begin{abstract}
Lipid vesicles immobilized via molecular linkers at a solid support represent a convenient platform for basic and applied studies of biological processes occurring at lipid membranes. Using total internal reflection fluorescence microscopy (TIRFM), one can track such processes at the level of individual vesicles provided that they contain dyes. In such experiments, it is desirable to determine the size of each vesicle, which may be in the range from 50 to $1000 \mathrm{~nm}$. Fortunately, TIRFM in combination with nanoparticle tracking analysis makes it possible to solve this problem as well. Herein, we present the formalism allowing one to interpret the TIRFM measurements of the latter category. The analysis is focused primarily on the case of unpolarized light. The specifics of the use of polarized light are also discussed. In addition, we show the expected difference in size distribution of suspended and immobilized vesicles under the assumption that the latter ones are deposited under diffusion-controlled conditions. In the experimental part of our work, we provide representative results, showing explicit advantages and some shortcomings of the use of TIRFM in the context under consideration, as well as how our refined formalism improves previously suggested approaches. C 2015 AIP Publishing LLC. [http://dx.doi.org/10.1063/1.4928083]
\end{abstract}

\section{INTRODUCTION}

One of the goals of applied physics is to provide a basis for design and fabrication of devises used to explore various processes. The corresponding physical background should be sufficient not only for the fabrication but also for the interpretation of the results of measurements. The latter depends on the properties of a subject of investigation and may often be far from straightforward. This is frequently the case in studies of biological processes taking place on the mesoscopic scale (from a few nm to $\mu \mathrm{m}$ ). A good example here is processes occurring on lipid membranes.

In cells, an appreciable fraction of proteins and many enzymes are associated with lipid membranes. ${ }^{1}$ For this reason, membranes play an important role in various inter- and intracellular processes, e.g., enzymatic reactions, ${ }^{1}$ contacts between cells, ${ }^{2}$ ion-, protein-, and vesicle-mediated cell-cell communication, ${ }^{3}$ and defence of cells against viruses and other pathogenes. ${ }^{4}$ The understanding of mechanisms and kinetics of such processes is obviously of interest from the viewpoints of biology and numerous applications in medicine and pharmacology. Compared to biophysical processes occurring in aqueous suspensions, membrane processes are also of interest from the perspectives of physics, because they are often complicated by various physical factors including formation of domains composed of different lipids and/or containing proteins, ${ }^{5}$ membrane curvature, ${ }^{6}$ and inherent and adsorbate-induced membrane strain. ${ }^{7}$ Despite high activity in this area, the progress is here still far from desirable. In vivo, the corresponding measurements are

\footnotetext{
a) Authors to whom correspondence should be addressed. Electronic addresses: zhdanov@catalysis.ru and fredrik.hook@chalmers.se
}

complicated by limited access of experimental tools to study membranes explicitly and also by the fact that membranes in cells are highly crowded. In vitro, the experiments are often performed with suspended vesicles at the ensemble level. ${ }^{1}$ The interpretation of such experiments is complicated by transport limitations and by the likely inherent and/or masstransport related dependence of the kinetics on vesicle size. Due to the latter factor, the observed kinetics represent a convolution of true kinetics and the vesicle size distribution (VSD), and accordingly, the true kinetics are in fact hidden. From the perspectives of theory, the analysis of membrane processes is challenging because the corresponding systems are heterogeneous on the mesoscopic scale.

A convenient alternative platform includes lipid bilayers and/or vesicles directly attached to or immobilized via linkers at a solid support. ${ }^{8}$ Such systems can be fabricated via adsorption of vesicles without or with subsequent spontaneous or peptide-induced rupture, ${ }^{8,9}$ or via the "solventassisted" procedure. ${ }^{10}$ The corresponding mass uptake can be determined optically by ellipsometry, waveguide lightmode spectroscopy and surface plasmon resonance, ${ }^{11-13}$ or by quartz crystal microbalance. ${ }^{12}$ Vesicles directly attached to a support are however deformed. To increase the control of the shape and size of vesicles, they can be immobilized at a support or supported lipid bilayer via links, e.g., by using NeutrAvidin. If the number of molecular tethers is kept low, the vesicle shape is expected to remain spherical, i.e., the membrane curvature is uniquely determined, and one can study its effect on various membrane processes as pioneered by Stamou and co-workers ${ }^{14}$ (for their methodology, see Ref. 15). Recent experiments of this category are focused at interaction of peptides and proteins with vesicles, ${ }^{14,16-18}$ 
function of single membrane-active enzymes, ${ }^{19-21}$ encapsulation efficiency, ${ }^{22}$ and vesicle docking and fusion. ${ }^{23}$ Alternatively, suspended vesicles may interact with various species attached to a supported lipid bilayer. ${ }^{24}$ In all these experiments, the kinetics were simultaneously tracked on a large number of individual fluorescent dye-labelled vesicles by using total internal reflection fluorescence microscopy (TIRFM) $^{17,20,21,24}$ or confocal fluorescence microscopy (CFM). ${ }^{14,15,19,22,23}$ (Historically, TIRFM was introduced to study various surface bioprocesses in the early $1980 \mathrm{~s} ;{ }^{25,26}$ for CFM and combination of TIRFM and CFM, see Refs. 27 and 28, respectively; the application of TIRFM and CFM to immobilized vesicles started in the early 2000s. ${ }^{29}$ )

In experiments with immobilized vesicles, their average size can be varied in a wide range from $50 \mathrm{~nm}$ to $1 \mu \mathrm{m}$, the dispersion of the size distribution is usually appreciable (the FWHM is comparable with the average), and accordingly, the characterization of vesicle sizes is an important step. In principle, the size distribution of vesicles can be measured in solution before immobilization by nanoparticle tracking analysis (NTA) ${ }^{30}$ or dynamic light scattering (DLS). ${ }^{31}$ This is efficient if one is interested in the scale of the average size and dispersion of size distribution. One of the reservations here is that attachment of vesicles is usually controlled by diffusion, the diffusion coefficient decreases with increasing vesicle size, and accordingly, the vesicle size distributions in solution and at the support are different. In addition, the measurements in solution do not allow one to determine the size of individual immobilized vesicles. The latter is, however, highly desirable in experiments with such vesicles. To measure the size of a vesicle, one can, in principle, employ AFM, SEM, or TEM. Vesicles are, however, flexible, and the accuracy of measurements of their size by AFM is limited. SEM and TEM analyses require drying and fixation steps, which significantly alters the vesicle structure. An additional serious problem is that one should use a large array of vesicles in order to study kinetics with high accuracy, and the identification of size of each vesicle in such arrays by AFM, SEM, or TEM is very impractical.

Fortunately, the fluorescence intensity of a vesicle depends on its size, and TIRFM and CFM make it possible not only to track kinetic processes on individual vesicles but also to determine their sizes, provided the dye concentration on the vesicle surface is sufficiently constant, irrespective of the vesicle radius (referring to the Poisson distribution, one can expect that this condition is met if the number of dye molecules per vesicle is appreciable, although the opposite has been reported for vesicles prepared with a low number of extrusion steps $^{32}$ ). The corresponding CFM- and TIRFMbased approaches were introduced, respectively, by Stamou and co-workers ${ }^{15}$ and in our work. ${ }^{20}$ The mathematical formalisms underlying these techniques are similar. Our formalism was, however, not described in detail. We just presented a single equation [Eq. (S1) in supplementary material, ${ }^{20}$ see also Eq. (17)] allowing us to interpret the results. Comparing our equation with that used in Ref. 15, one can notice that some of the terms which are expected to be identical are in fact different, and that our equation is in agreement with that derived earlier by Lee $e t a l .{ }^{33}$ for the shell model in the
TIRFM context (we were not aware of the latter work, and it was not mentioned in Ref. 20).

Herein, we present in detail the formalism allowing one to correctly use TIRFM (as well as CFM) for determination of size of individual vesicles (Sec. II) and illustrate experimentally its application (Sec. III). For unpolarized light, our analysis includes the derivation of the equation used earlier ${ }^{20}$ and identifies that the above-mentioned discrepancy between Refs. 20 and 15 is related to a mathematical error in Ref. 15. In addition, we scrutinize the case of polarized light and predict what can be obtained here (these results form a basis for experiments complementary to those based on fluorescence polarization microscopy ${ }^{34}$ ). On the ensemble level, we illustrate the difference between the size distributions of suspended and immobilized lipid vesicles. Our complementary representative experiments show advantages and some shortcomings of the use of TIRFM in the context under consideration.

\section{THEORETICAL BACKGROUND}

\section{A. Excitation of randomly oriented dyes}

In TIRFM (or CFM) experiments with vesicles immobilized at the interface as schematically shown in Fig. 1(a), one measures fluorescence intensity, $I$, of dye molecules located at single vesicles and being excited by the evanescent field. To calculate the dependence of $I$ on the vesicle size, we consider that vesicles are spherical and that the dye molecules are attached to both (external and internal) leaflets of surface-immobilized vesicles. In principle, dyes may be attached to one of the leaflets, e.g., to the external leaflet. The difference between these two cases is related to the difference of the radii of the external and internal leaflets and also to different dye orientations there. In our calculations, the former factor is neglected because the thickness of a lipid layer is typically small compared to the vesicle radius. The latter factor is expected to be negligible, because even in the case of preferable orientation, the orientation vectors on both sides are anyway similar, and accordingly, the light absorption at a given coordinate will be the same.

The dye distribution at the leaflets is assumed to be uniform, and the dye concentration (per unit area) to be the same for all vesicles irrespective of their radius $r$ (the inevitable fluctuations of the dye population compared to the expected average value can, in principle, be measured ${ }^{32}$ and taken into account as the second-order correction; this is beyond our present goals). In this case, the observed fluorescence intensity, $I(r)$, of a vesicle is proportional to an integral of the light-absorption rate over the vesicle surface. In general, $I(r)$ depends on the light polarization and dye orientation with respect to the vesicle leaflet. The simplest case is realized when the dye molecules are flexibly linked to the leaflets of a vesicle and, due to different orientations related to rotational diffusion, their absorbance is on average independent of the light polarization. In this case, $I(r)$ can be calculated, taking into account only the decrease of the evanescent field with increasing the coordinate, $z$, perpendicular to the glass-solution interface (see Appendix A), i.e., 


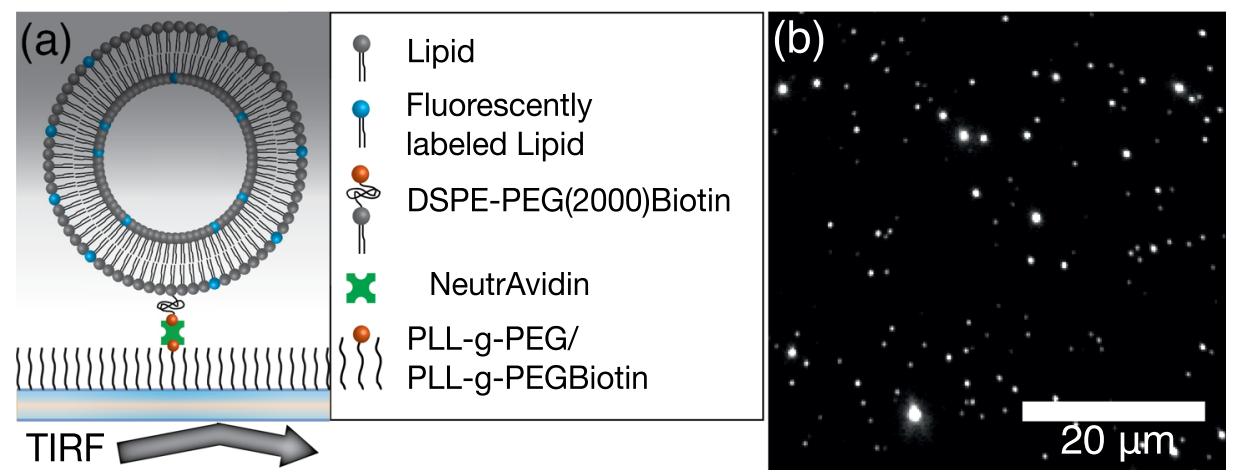

FIG. 1. (a) Scheme of surface modification and immobilization of vesicles using biotin-NeutrAvidin linking. The substrate was modified with PLL-g-PEG/ PLL-g-PEGbioin for specific binding of Neutravidin ensuring in turn specific binding of vesicles containing biotin-modified lipids (DSPE-PEG(2000)Biotin) in addition to unlabeled and fluorescently labeled lipids. Increasing darkness of the background illustrates decrease of the evanescent field. The arrow shows schematically incoming and outcoming light. Its reflection takes place at the glass-solution interface. (b) TIRFM-micrograph showing vesicles immobilized on the PLL-g-PEG/PLL-g-PEGBiotin support for the polydisperse sample.

$$
I(r)=A J_{0} \int \exp (-z / \delta) d s
$$

where $J_{0}$ is the intensity of the incident light, $A$ is a constant proportional to the dye concentration and absorption cross section, $\delta$ is the extinction length, and the integration is performed over the vesicle surface ( $d s$ is an element of the surface).

The use of (1) implies that the perturbation of the exponential evanescent field due to the presence of immobilized species is negligible. The experiments focused on this aspect $^{35}$ indicate that this is often the case. For vesicles in general and especially for sparsely immobilized vesicles, this approximation is expected to be very good, because the lipids occupy only a tiny part of the space near the support. In addition, the effect of the support on the light emitted by a dye after excitation is considered to be negligible as well. Concerning the latter approximation, one should bear in mind that the size of vesicles is relatively large on the wavelength scale. Thus, the emission of dyes located only in the vesicle nearest to the glass/water interface could be influenced by the substrate. In fact, however, the effect of this interface is negligible even in this region, because the tether is usually rather long (e.g., $15-17 \mathrm{~nm}$ in our case).

To calculate integral (1) and similar integrals below, one can use either the Cartesian coordinates along, $x$ and $y$, and perpendicular, $z$, to the interface (with $z=0$ corresponding to the bottom of a vesicle), or the spherical coordinates including the radial coordinate, $r$, identified with the vesicle radius, and two angular coordinates, $0 \leq \theta \leq \pi$ and $0 \leq \phi \leq 2 \pi$. In our calculations, we consider that $\theta=0$ corresponds to the top of a vesicle and employs the following relation between the Cartesian and spherical coordinates:

$$
x=r \sin \theta \cos \phi, \quad y=r \sin \theta \sin \phi, \quad z=r(\cos \theta+1) .
$$

In the spherical coordinates, which are more convenient, an element of the external vesicle surface area is given by $d s=r^{2} \sin \theta d \theta d \phi$, and expression (1) can be rewritten as

$$
I(r)=A J_{0} r^{2} \int_{0}^{2 \pi} \int_{0}^{\pi} \exp [-r(\cos \theta+1) / \delta] \sin \theta d \theta d \phi .
$$

Then, the elementary integration yields

$$
\begin{gathered}
I(r)=2 \pi A J_{0} r \delta[1-\exp (-2 r / \delta)], \quad \text { or } \\
I(r)=4 \pi A J_{0} r^{2} \quad \text { at } \quad \delta \gg r, \quad \text { and } \\
I(r)=2 \pi A J_{0} r \delta \quad \text { at } \delta \ll r .
\end{gathered}
$$

\section{B. Excitation of specifically oriented dyes}

If light is polarized and the dye orientation with respect to the vesicle surface is fixed (for examples of specifically oriented dyes, see Ref. 36), its absorption depends not only on $z$ but also on $\theta$ and $\phi$. Let us first consider the situation when light is $s$-polarized and a dye is excited only by the component of the electric field perpendicular to the vesicle surface. To perform calculations, we assume that the plane of incidence includes the $x$ and $z$ axes. The electric field will in this case be oriented along the $y$ axis (Appendix A). The $y$ component of the unit vector oriented perpendicular to the vesicle surface is $\sin \theta \sin \phi$. The light absorption cross section is proportional to the square of this component, i.e., to $\sin ^{2} \theta \sin ^{2} \phi$. Taking this factor into account during integration [cf. Eq. (2)], we have

$$
\begin{aligned}
I(r)= & \left.A J_{0} r^{2} \int_{0}^{2 \pi} \int_{0}^{\pi} \exp [-r(\cos \theta+1) / \delta)\right] \sin ^{2} \theta \sin ^{2} \phi \\
& \times \sin \theta d \theta d \phi,
\end{aligned}
$$

and after integration over $\phi$

$$
\begin{gathered}
\left.I(r)=\pi A J_{0} r^{2} \int_{0}^{\pi} \exp [-r(\cos \theta+1) / \delta)\right] \sin ^{3} \theta d \theta, \quad \text { or } \\
I(r)=(4 \pi / 3) A J_{0} r^{2} \quad \text { at } \quad \delta \gg r, \quad \text { and } \\
I(r)=2 \pi A J_{0} \delta^{2} \quad \text { at } \delta \ll r .
\end{gathered}
$$

[Note that for $s$-polarization the excitation cross section is proportional to $E_{2 y}(0)^{2}$, while $J_{0}$ is proportional to $E_{0 y}^{2}$ (for the designations, see Appendix A). In the equations presented here, the difference between $E_{2 y}(0)$ and $E_{0 y}$ is included into $A$ in order to reduce their size. The situation 
with the equations outlined in the paragraph below is similar.]

The dependence of the TIRFM intensity on the vesicle radius can also be easily calculated for other orientations of dyes and/or for $p$-polarized of light. If, for example, dyes are oriented as described above, light is $p$-polarized, and $\sin \theta_{0}$ is slightly above $n_{2} / n_{1}$, the electric field is oriented primarily along the $z$ axis (see Appendix A), the $z$ component of the unit vector oriented perpendicular to the vesicle surface is $\cos \theta$, and accordingly, the light absorption cross section will be proportional to $\cos ^{2} \theta$. Taking this into account during integration [cf. Eq. (2)], we obtain

$$
\left.I(r)=A J_{0} r^{2} \int_{0}^{2 \pi} \int_{0}^{\pi} \exp [-r(\cos \theta+1) / \delta)\right] \cos ^{2} \theta \sin \theta d \theta d \phi,
$$

and, after integration over $\phi$

$$
\begin{gathered}
\left.I(r)=2 \pi A J_{0} r^{2} \int_{0}^{\pi} \exp [-r(\cos \theta+1) / \delta)\right] \cos ^{2} \theta \sin \theta d \theta, \quad \text { or } \\
I(r)=(4 \pi / 3) A J_{0} r^{2} \text { at } \delta \gg r, \text { and } \\
I(r)=2 \pi A J_{0} r \delta \text { at } \delta \ll r .
\end{gathered}
$$

Expression (12) is similar to (4) and (8), while (13) is similar to (5) and different compared to (9). In particular, the intensity predicted by (13) is much larger than that predicted by (9). Thus, the use of different light polarizations may help to clarify the dye orientation with respect to the vesicle leaflets provided $\delta \ll r$.

If dyes are oriented randomly along the vesicle surface and light is $s$ - or $p$-polarized, one should just replace $\sin ^{2} \theta \sin ^{2} \phi$ by $1-\sin ^{2} \theta \sin ^{2} \phi$ in Eq. (6) or replace $\cos ^{2} \theta$ by $\sin ^{2} \theta$ in Eq. (10), respectively.

In fact, expressions (8) and (12), obtained at $\delta \gg r$, provided a dye is excited only by the component of the electric field perpendicular to the vesicle surface, are identical. In this limit, the electric field is nearly the same in all the regions of a vesicle, and after averaging of the absorption cross section over the dipole orientation in different regions, the final results are independent of the light polarization and dipole orientation. If $\delta \ll r$, the fluorescence takes place primarily near the bottom of a vesicle and accordingly strongly depends on light polarization.

The equations derived above identify the relation between $I$ and $r$ in various situations. The coefficient of proportionality, $A$, employed there cannot be accurately calculated. In the application of these equations, this coefficient can be considered as a free or fitting parameter. Practically, this means that in the absence of additional information, the equations derived allow one to obtain only ratios of size of vesicles and the shape of the vesicle size distribution but not the size values. To get the size values, one can use the vesicle size distribution measured in the bulk of solution by NTA (or DLS). As already noticed in the Introduction, the size distributions in the bulk and on the surface are different, but the difference is usually not appreciable (see Sec. IIE).
Thus, one can, in principle, identify the vesicles with the most probable size in the bulk with those with the most probable size on the surface. A more accurate approach is to correct the measured NTA distribution by taking the specifics of vesicle diffusion into account (see Sec. IIE) and to use the maximum of the corrected NTA distribution for size identification. These procedures make it possible to determine size of each immobilized vesicle.

\section{Other general relations}

Our analysis above corresponds to TIRFM. The dye excitation under the CFM conditions can be described in analogy by replacing the exponential intensity profile by a suitable one. As already mentioned in the Introduction, the corresponding application of CFM was described by Kunding et al. ${ }^{15}$ In their analysis of dye excitation at a vesicle, the emitted fluorescence signal is expressed via an integral along the $z$ axis as [see Eqs. (3) and (4) in Ref. 15 and note that we use slightly different designations in order to unify our presentation]

$$
I(r) \simeq A J_{0} \int_{0}^{2 r} p(z) G(r, z) d z
$$

where $p(z)$ is the light-intensity distribution, and

$$
G(r, z) d z \equiv 2 \pi\left(2 r z-z^{2}\right)^{1 / 2} d z
$$

is an element of the external vesicle surface area corresponding to the vertical coordinate from $z$ to $z+d z$ (Fig. 3).

$G(r, z) d z$ can be calculated by using the spherical coordinates, i.e., representing an element of the external vesicle surface area as $d s=r^{2} \sin \theta d \theta d \phi$, integrating over $\phi$ from 0 to $2 \pi$, and taking into account that $d z=r \sin \theta d \theta$. This procedure yields

$$
G(r, z) d z \equiv 2 \pi r d z
$$

Substituting (16) into (14) and taking into account that in our (TIRFM) context $p(z)=\exp (-z / \delta)$, we have

$$
\begin{aligned}
I(r) & =A J_{0} \int_{0}^{2 r} \exp (-z / \delta) 2 \pi r d z \\
& =2 \pi A J_{0} r \delta[1-\exp (-2 r / \delta)] .
\end{aligned}
$$

This expression is equivalent to (3). In slightly different designations, it was employed in our previous work ${ }^{20}$ where we used the Cartesian coordinates. A similar equation can also be found in Ref. 33 .

Comparing expressions (15) and (16), one can notice that they are different. To identify the source of the difference, it is convenient to introduce radius $\rho \equiv\left(2 r z-z^{2}\right)^{1 / 2}$ corresponding to a given value of $z$ (Fig. 2). $G(r, z) d z$ can then be represented as

$$
G(r, z) d z=2 \pi \rho d l
$$

where $d l$ is a length element defined as a distance between points $B$ and $C$ (Fig. 2). An elementary geometrical analysis 


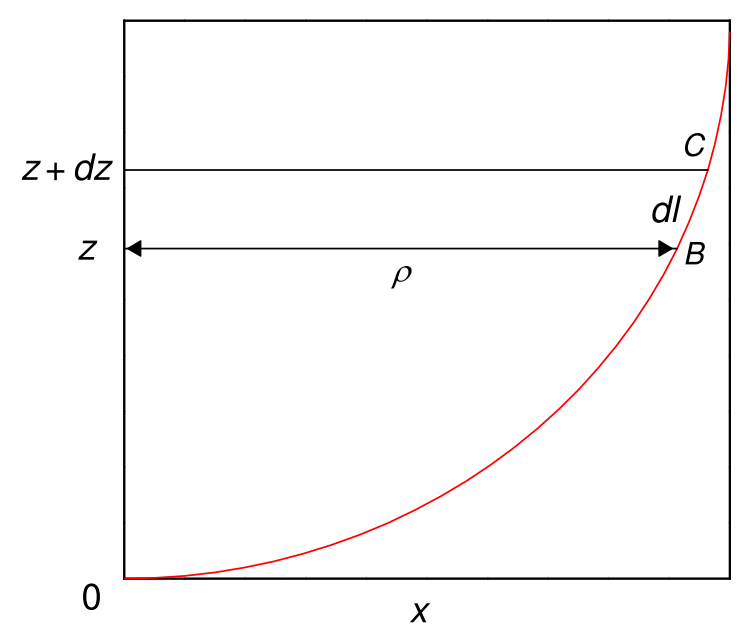

FIG. 2. Fragment of a vesicle cross section for illustration of calculation of an element of the external vesicle surface area corresponding to the vertical coordinate from $z$ to $z+d z$.

yields $d l=r d z / \rho$. Substituting this relation into (18), we obtain expression (16). Expression (15) used by Stamou et $a l .{ }^{15}$ can be obtained as

$$
G(r, z) d z=2 \pi \rho d z=2 \pi\left(2 r z-z^{2}\right)^{1 / 2} d z .
$$

This expression corresponds, obviously, to a vertically oriented cylinder with radius $\rho$. The surface of the shell is, however, tilted and cannot be replaced by a cylinder (Fig. 2). For this reason, strictly speaking, expressions (15) and (19) are incorrect.
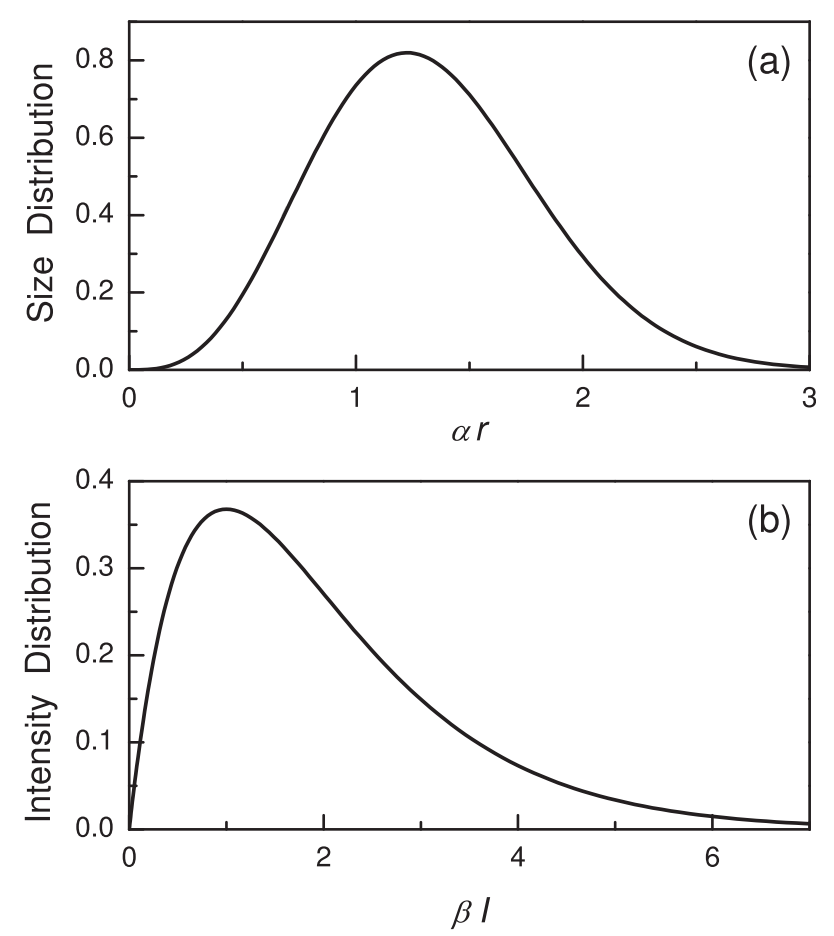

FIG. 3. (a) Size distribution of immobilized vesicles and (b) the corresponding TIRFM intensity distribution. The former distribution is chosen, for example, as $F(r)=2 \alpha^{4} r^{3} \exp \left(-\alpha^{2} r^{2}\right)$, where $\alpha$ is a parameter. The latter distribution was calculated by using Eqs. (23) and (4) and shown by employing the dimensionless variable, $\beta I$, where $\beta \equiv \alpha^{2} /(2 \pi A J)$.
To illustrate explicitly the limits of applicability of Eqs. (14) and (15), we use them in our context, i.e., with $p(z)=\exp (-z / \delta)$. This yields

$$
\begin{gathered}
I(r)=2 \pi A J_{0} \int_{0}^{2 r}\left(2 r z-z^{2}\right)^{1 / 2} \exp (-z / \delta) d z, \quad \text { or } \\
I(r)=\pi^{2} A J_{0} r^{2} \quad \text { at } \delta \gg r, \quad \text { and } \\
I(r)=2^{1 / 2} \pi^{3 / 2} A J_{0} r^{1 / 2} \delta^{3 / 2} \text { at } \delta \ll r .
\end{gathered}
$$

Comparing expressions (21) and (4) obtained for relatively small vesicles with $r \ll \delta$, one can notice that they are similar, and the difference is only in a numerical factor. This difference is insignificant, because the numerical factor can be included into $A$. In contrast, expressions (22) and (5) predict different dependences of $I$ or $r\left(\propto r^{1 / 2}\right.$ and $r$, respectively). Thus, Eq. (14) in combination with Eq. (15) [or (19)] is inaccurate for relatively large vesicles with $r>\delta$.

\section{Intensity and size distributions of immobilized vesicles}

Measuring the intensities of single immobilized vesicles, one can construct the fluorescence intensity distributions, $f(I)$. If the dependence of the intensity on size, $I(r)$, is known, one can also construct the size distribution, $F(r)$, of these vesicles by using the same experimental data. The relationship between these two distributions can be obtained by employing the conventional rule of the change of variables

$$
f(I)=F(r(I)) \frac{d r(I)}{d I},
$$

where $r(I)$ is the function inverse to $I(r)$.

Our analysis above shows that the dependence of $I$ on $r$ can be linear [see, e.g., (5)]. For an ensemble of vesicles, this means that the shape of the distributions $f(I)$ and $F(r)$ [Eq. (23)] will be the same in this case. In the opposite limit (4), the shapes are different as shown in Fig. 3 where the size distribution is, for example, chosen to be $F(r)$ $=2 \alpha^{4} r^{3} \exp \left(-\alpha^{2} r^{2}\right)$, where $\alpha$ is a parameter inversely proportional to the average size.

\section{E. Size distributions of suspended and immobilized vesicles}

As already noted in the Introduction, the attachment of vesicles to a substrate is usually controlled by diffusion in solution, the hydrodynamic diffusion coefficient decreases with increasing vesicle size, $D \propto 1 / r$, and accordingly, the vesicle size distributions in solution, $\mathcal{F}(r)$, and at the interface, $F(r)$, are different. The relation between these size distributions is determined by the flow conditions in a measurement cell. Two generic situations are realized when the flow is negligible and appreciable, respectively. In the no-flow case, the diffusion flux of monodisperse vesicles is well known to be e $^{37}$

$$
J=\left(\frac{D}{\pi t}\right)^{1 / 2} c
$$


where $t$ is time, and $c$ is the vesicle concentration in solution. For appreciable flow along the cell (of length $L$ ) with a rectangular cross section (with sizes $h \ll l \ll L$ ), the diffusion flux of monodisperse vesicles towards the $l \times L$ wall is given in Ref. 38 (see also Ref. 39 for the extended treatment)

$$
J=0.97\left(\frac{v_{\circ} D^{2}}{h x}\right)^{1 / 3} c
$$

where $v_{\circ}$ is the average flow velocity, and $x(0 \leq x \leq L)$ is the coordinate along the channel. Taking the dependence of $D$ on $r$ into account, flaxes (24) and (25) can be represented as $J \propto c / r^{\beta}$, where $\beta=1 / 2$ and $2 / 3$ in the former and latter cases, respectively. If vesicles in solution are distributed over size, the latter expression can be used for vesicles of each size. This means that the size distribution of immobilized vesicles, $\mathcal{F}(r)$, is proportional to $F(r)$ and $1 / r^{\beta}$, i.e.,

$$
\mathcal{F}(r)=r^{-\beta} F(r) / \int_{r_{\min }}^{r_{\max }} r^{-\beta} F(r) d r,
$$

where $r_{\min }$ and $r_{\max }$ are the minimum and maximum radii. For example, let us consider that the vesicle size distribution in solution is given by $F(r)=2 \alpha^{4} r^{3} \exp \left(-\alpha^{2} r^{2}\right)$, where $\alpha$ is a parameter. The two corresponding distributions $\mathcal{F}(r)$ calculated according to (26) with $\beta=1 / 2$ and $2 / 3$, respectively, are shown in Fig. 4 together with the bulk distribution. The shapes of the three distributions are seen to be nearly identical. The surface distributions are, however, slightly shifted to smaller $r$, because the corresponding vesicles diffuse faster towards the surface, and their contribution to the size distribution in the adsorbed state is enhanced. In particular, the shift of the position of the maximum is $\simeq 15 \%$, which is instructive for studies of this type.

\section{EXPERIMENTAL RESULTS AND DISCUSSION}

Our TIRFM experiments with individual vesicles were designed to demonstrate the difference in VSDs obtained using Eqs. (3) and (20). The so-determined VSDs were compared with those obtained for the same vesicle preparation by employing NTA. Two different vesicle samples were

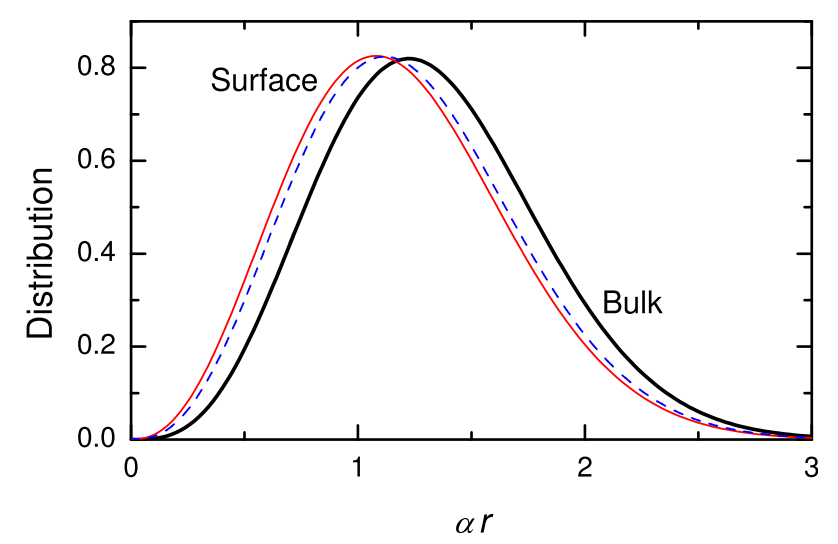

FIG. 4. Size distribution of vesicles in the bulk of solution (thick solid line) and the two corresponding size distributions of vesicles immobilized on the surface. The latter two distributions were calculated by employing (26) with $\beta=1 / 2$ (dashed line) and 2/3 (thin solid line), respectively. produced as generic examples, one with monodisperse vesicles (mean radius $\simeq 104 \mathrm{~nm}$ ), representing the most commonly used system in biophysical studies, and one with a bimodal size distribution and significantly larger mean radius. The corresponding methodological aspects are described in Appendix B.

Vesicles were immobilized as schematically illustrated in Fig. 1, together with a representative TIRFM micrograph of vesicles of the polydisperse sample. As seen in the micrograph, the vesicle coverage $\left(\sim 110\right.$ vesicles per $\left.50 \times 50 \mu \mathrm{m}^{2}\right)$ corresponds to a mean vesicle-to-vesicle distance of $\sim 5 \mu \mathrm{m}$ for the polydisperse sample. Together with the low biotin coverage, this ensures that each bright dot corresponds to a single lipid vesicle (under such conditions, the probability that two or more vesicles conjugate together and are tracked as one bright dot is negligibly low). To generate sufficient statistics for determination of the VSDs, 19 micrographs taken from different regions on the same substrate were analyzed $\left(\sim 9 \times 10^{5} \mu \mathrm{m}^{2}\right)$.

The VSDs obtained from TIRFM micrographs of the monodisperse vesicles by using Eqs. (3) and (20) are shown in Fig. 5(a) together with those determined employing NTA. The corresponding results for the vesicle sample with a bimodal VSD are presented in Fig. 5(b). For both samples, the factor $A J_{0}$ in Eq. (3) or (20) was chosen so that the positions of the maxima in the TIRFM and NTA distributions coincide (as earlier suggested in the literature ${ }^{15,40}$ ).

One rarely considered factor that complicates the direct comparison between NTA measurements made in bulk and TIRFM measurements performed on a surface is that diffusion limitations promote adsorption of smaller sized vesicles (Sec. II E). The NTA VSDs shown in Fig. 5 take this discrepancy into account. They were obtained by converting the bulk NTA VSDs via Eq. (26) with $\beta=2 / 3$ that corresponds to laminar flow conditions (the VSD calculated with $\beta=$ $1 / 2$ was nearly the same). This correction improves the accuracy of the results.

The results shown in Fig. 5(a) demonstrate that the two TIRFM VSDs determined by Eqs. (3) and (20) agree very well with the NTA VSD up to $r=140 \mathrm{~nm}$, while in both cases there is a significant deviation from the NTA VSD with $r$ increasing above $140 \mathrm{~nm}$. Regarding this discrepancy, which is commonly observed in corresponding measurements, ${ }^{40}$ it is worth recalling that the determination of the NTA VSDs is based on the dependence of the diffusion constant on vesicle radius, while in contrast, the TIRFM VSDs are based on the total integrated fluorescence intensity originating from the presence of fluorescent lipids in the lipid bilayer of the vesicles. The overestimation of the number of vesicles at large $r$ in the TIRFM VSDs is therefore likely due to the presence of a non-negligible fraction of multilamellar vesicles $(\mathrm{MLVs})^{40}$ (such vesicles influence, e.g., the experiments with membrane-active peptides ${ }^{41}$ ). Although vesicle production based on rehydration of thin lipid films is known to partly produce $\mathrm{MLVs}_{\mathrm{s}}{ }^{42}$ treatment of such MLVs with multiple extrusion and freeze-thawing steps, as done here, has been reported to reduce the fraction of MLVs. ${ }^{43}$ Our results suggest, however, that there was still a measurable fraction of MLVs present in both the monodisperse 
(a)

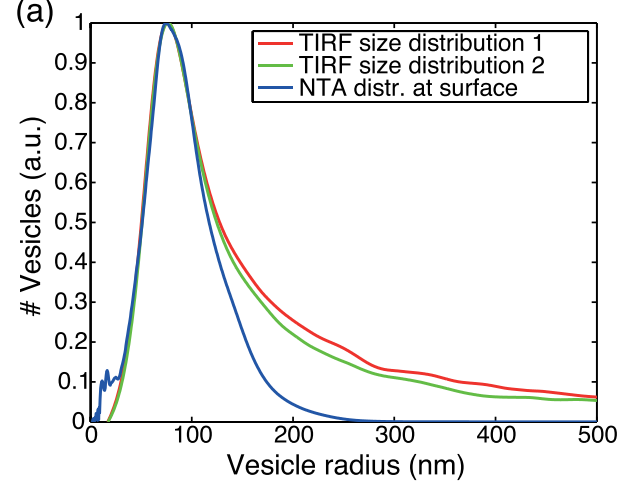

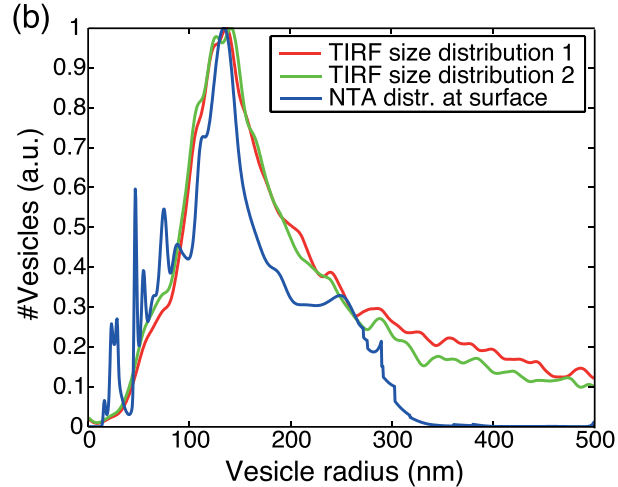

FIG. 5. (a) TIRFM size distributions 1 and 2 of monodisperse lipid vesicles obtained, respectively, by using Eqs. (3) and (20) with $\delta=100 \mathrm{~nm}$. For comparison, the NTA distribution is calculated taking diffusion-related corrections into account is also shown. (b) As (a) for vesicles with a bimodal size distribution.
[Fig. 5(a)], which was exposed to multiple freeze thawing steps, and the bimodal [Fig. 5(b)] sample. In the latter case, however, the existence of an appreciable fraction of larger vesicles, as verified from the NTA results, appears to contribute significantly to the shape of the TIRFM VSDs with increasing $r$.

In principle, the aggregation of dyes and/or a broad distribution of the dye numbers in vesicles of equal sizes (as discussed in Ref. 32) cannot be fully excluded either. Such effects are expected to result in a much larger value of FWHM in the TIRFM VSDs compared to that in the NTA case. In our situations, however, the FWHMs are either nearly equal (Fig. 5(a)) or the TIRFM FWHM is somewhat larger but not dramatically larger. In addition, the NTA results can be influenced by fluctuations of the shape of vesicles. The vesicles under consideration are, however, relatively small and appreciable deviation of their shape from spherical are not likely. Concerning this aspect, the comparison of the NTA data with direct measurement from electron micrographs shows that NTA at least does not underestimate the fraction of large vesicles. ${ }^{44}$ Thus, the difference between the TIRFM and NTA results can hardly be attributed to the shortcomings of NTA.

From the analysis of the results shown in Fig. 5, it appears as if the major influence on the discrepancy between the NTA and TIRFM VSDs originates from the existence of multilamellar vesicles, rather than the difference between the commonly applied Eq. (20) and the herein derived Eq. (3). In fact, only minor differences between the VSDs determined using Eqs. (3) and (20) were observed at vesicle radii significantly larger than the corresponding mean radii. However, the most significant impact of Eqs. (3) and (20) is not on how the actual size distributions compare, but rather on the error introduced when the intensity measured for an individual vesicle is converted into its radius. This is shown in Fig. 6(a), which illustrates that there is indeed no significant difference in the size determination $(<4 \%)$ using the two expressions up to $r$ smaller than around $140 \mathrm{~nm}$. However, already for vesicles with the intensity 1.5 times larger than that of a $140 \mathrm{~nm}$ vesicle, the error is $31 \%$, and increases to $95 \%$ for vesicles with 3 times larger intensity.

As shown in Fig. 6(b), an appreciable fraction ( 48\%) of the binomially size distributed vesicles has intensities that cause errors larger than $30 \%$ when their individual intensity levels are converted into vesicle size (the corresponding fraction for the monodisperse sample was $\sim 8 \%$ (not shown)). Such a large error in the size determination might significantly influence conclusions drawn on the dependence of different biophysical properties on vesicle size, thus suggesting that the previous work using CFM and basing the analysis on Eq. (15) might benefit from having the analysis revisited.

When Eq. (14) in combination with (15) [or (20) in our context] was initially used to describe how the fluorescence intensity from immobilized lipid vesicles depends on vesicle size, ${ }^{15}$ it was proposed to be valid for vesicles with a radius up to $\sim 500 \mathrm{~nm}$, while nanometer precision was later claimed hold for vesicles up to $350 \mathrm{~nm}$. ${ }^{45}$ Our analysis indicates that the sizes determined using Eqs. (3) and (20) start to deviate
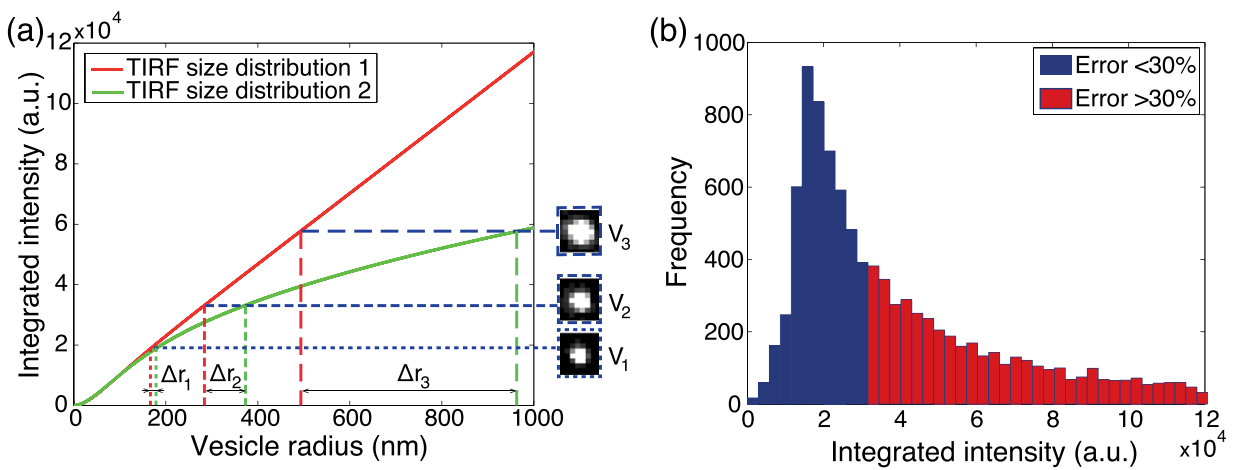

FIG. 6. (a) Intensity to radius relations 1 and 2 [Eqs. (3) (size distribution 1) and (20) (size distribution 2) with $\delta=100 \mathrm{~nm}$ ] constructed by using the prefactors $\left(A J_{0}\right)$ determined for the polydisperse sample (Fig. $5(\mathrm{~b})$ ). Three vesicles with different integrated intensities (micrographs of $\left.1.6 \times 1.8 \mu \mathrm{m}^{2}\right)$ were employed to illustrate how the difference in radius estimation from Eqs. (3) and (20) increases rapidly with increasing vesicle intensity. (b) Frequency of TIRFM intensities calculated by using Eq. (3) for all recorded polydisperse vesicles. The regions where Eq. (20) results in the error smaller and larger than $30 \%$ are indicated by different colours. 
when the vesicle radius approaches the penetration depth of the evanescent wave $(r \simeq \delta)$. It is important to stress in this context that we have applied this analysis for TIRFM-based illumination, where the penetration depth is $\sim 100 \mathrm{~nm} .^{46}$ CFM, which was used in the majority of the papers in the field, including Refs. 14, 16, and 45, and can also be characterized with a decaying excitation intensity, although extending up to $\sim 500-600 \mathrm{~nm} .^{46,47}$ This suggests that the range of accuracy reported (up to $500 \mathrm{~nm}$ using confocal microscopy) is within the boundary for discrepancy we observed between size estimations using (3) and (20). Although strongly surface-confined illumination used (e.g., in studies requiring elimination of bulk fluorescence and improved signal to noise ratio) is more critical, it remains preferable to employ physically and mathematically correct representations when experimental data are being analyzed, which is particularly true in cases when the incorrect Eq. (15) was applied in situations with relatively short decay length.

\section{CONCLUSION}

The use of TIRFM for studies of the biophysical and chemical processes occurring at lipid vesicles immobilized at a solid support with simultaneous determination of the size of each individual vesicle are still not numerous (see the Introduction). Our present contribution to this field can be summarized as follows:

(i) We have derived general equations (Sec. II) helping to guide, perform, and interpret such studies. The analysis has been focused primarily on the case of unpolarized light. Application of the corresponding theoretical results has been illustrated experimentally in two generic situations (Sec. III). For a monodisperse vesicle sample with relatively small radii, the TIRFM VSDs obtained by using our Eqs. (3) and (20), derived by employing the prescription from Ref. 15, are found to be nearly identical. For a heterogeneous vesicle sample with larger sizes, on the other hand, there is a significant difference in vesicle sizes predicted by using Eqs. (3) and (20). This means that the conclusions drawn in the literature, by employing CFM (or TIRFM) based on the formalism from Ref. 15, may have to be revisited (it may change some of the reported quantitative results). Comparing the TIRFM and NTA, VSDs have shown that the former typically exhibits a large-size tail, which is likely due to the presence of a non-negligible fraction of multilamellar vesicles. This observation is also instructive for the understanding of the limits of applicability of the TIRFM and CFM VSDs.

(ii) The equations for polarized light have been derived as well. In particular, we have shown that the dye orientation with respect to the membrane surface can be determined by using light of different polarizations, provided $\delta / r \ll 1$. This opens up opportunities for obtaining qualitatively new results in the field under consideration.

(iii) The relations between the VSDs in the bulk of solution and in the immobilized state have been identified.
For example, the latter VSD of immobilized vesicle size is found to be shifted by $\simeq 15 \%$ compared to the former one.

Taking into account that the interest in lipid-membrane processes and in the corresponding applications of TIRFM and CFM (concerning the applications, see reviews ${ }^{48}$ and original studies ${ }^{49}$ in addition to numerous already mentioned references) is now high and expected to increase, we believe that the results presented will be useful in many different contexts.

\section{ACKNOWLEDGMENTS}

We thank Stephan Block for sharing with us his expertise in TIRFM. This work was supported by Swedish Research Council.

\section{APPENDIX A: EVANESCENT WAVE}

In TIRFM, the total reflection of light propagating in medium 1 (support) occurs from the optically less dense medium 2 (liquid with $n_{2}<n_{1}$ ) at $\sin \theta_{0}>n_{2} / n_{1}$, where $\theta_{0}$ is the incidence angle, and $n_{1}$ and $n_{2}$ are the corresponding refractive indices. The dye molecules, located in medium 2 at or near the interface between the media, are excited by the evanescent field. Its intensity decays exponentially

$$
J_{2}(z) \propto J_{0} \exp (-z / \delta),
$$

where $\delta=1 /\left[(2 \omega / c)\left(n_{1}^{2} \sin ^{2} \theta_{0}-n_{2}^{2}\right)^{1 / 2}\right]$ is the extinction length, $J_{0}$ is the intensity of the incident light, and $\omega$ is the light frequency.

The equation above forms a basis for the analysis of the excitation of dye provided that the measured excitation rate is independent of light polarization (in our context, this is the case if, e.g., the dye molecules have random orientations with respect to the membrane). In general, however, the light polarization should be taken into account. The corresponding equations can be found in textbooks (e.g., Ref. 50) or reviews (e.g., Ref. 26). To be specific, let us consider that the plane of incidence includes the $x$ and $z$ axes, and light is $s$-polarized. In this case, the electric field is oriented along the $y$ axis, the evanescent field is described as

$$
E_{2 y}^{2}(z)=E_{2 y}^{2}(0) \exp (-z / \delta),
$$

and the amplitudes of the field in the two media are related as

$$
E_{2 y}(0)=\frac{2 n_{1} \cos \theta_{0}}{\left(n_{1}^{2}-n_{2}^{2}\right)^{1 / 2}} E_{0 y} .
$$

(The phases are not important in our context.)

If light is $p$-polarized, there are two components of the electric field oriented, respectively, along the $x$ and $z$ axis. In particular, the evanescent field behaves as

$$
\begin{aligned}
& E_{2 x}^{2}(z)=E_{2 x}^{2}(0) \exp (-z / \delta), \\
& E_{2 z}^{2}(z)=E_{2 z}^{2}(0) \exp (-z / \delta),
\end{aligned}
$$


and at the interface the components of the electric filed are expressed via the amplitude of the field in the incident wave as

$$
\begin{aligned}
E_{0 x} & =E_{0} \cos \theta_{0}, E_{0 z}=E_{0} \sin \theta_{0}, \\
E_{2 x}(0) & =\frac{2 \cos \theta_{0} n_{1}\left(n_{1}^{2} \sin ^{2} \theta_{0}-n_{2}^{2}\right)^{1 / 2}}{\left(n_{2}^{4} \cos ^{2} \theta_{0}+n_{1}^{4} \sin ^{2} \theta_{0}-n_{1}^{2} n_{2}^{2}\right)^{1 / 2}} E_{0}, \\
E_{2 z}(0) & =\frac{2 \sin \theta_{0} \cos \theta_{0} n_{1}^{2}}{\left(n_{2}^{4} \cos ^{2} \theta_{0}+n_{1}^{4} \sin ^{2} \theta_{0}-n_{1}^{2} n_{2}^{2}\right)^{1 / 2}} E_{0} .
\end{aligned}
$$

\section{APPENDIX B: MATERIAL AND METHODS}

\section{Vesicle preparation}

The sample with monodisperse vesicles was made of POPC lipids, fluorescently labeled DOPE-ATTO647N (ATTO-TEC, Germany), and DSPE-PEG(2000) Biotin lipids at a ratio of (99:1:0.0023, w/w/mol. \%). The sample with bimodal vesicle distribution was made using DMPC, POPG, Cholesterol, DOPE-ATTO647N (ATTO-TEC, Germany), and DSPE-PEG(2000) Biotin lipids at a ratio of (69:25:5:1:0.0023,w/w/w/w/mol. \%); all lipids from Avanti Polar Lipids (USA) unless otherwise stated. The bimodal distribution was made through extrusion of giant unilamellar vesicles (GUVs). DMPC lipids are commonly used to produce GUVs, and it was found that by including a fraction of charged lipids and cholesterol, the number of daughter vesicles, and/or multilamellarity was reduced. ${ }^{51}$

Larger vesicles with bimodal size distribution were produced by a combination of two protocols designed for making GUVs based on rehydration in the presence of sucrose $^{51}$ In brief, $2.4 \mathrm{mg}$ of the lipid mixture dissolved in chloroform (Sigma-Aldrich, Germany) was dried under $\mathrm{N}_{2^{-}}$ gas exposure into a thin lipid film on the bottom of a round flask that was subsequently evacuated for about an hour to remove remaining traces of the solvent. The lipid film was then rehydrated with $2.4 \mathrm{ml}$ of a $0.5 \mathrm{M}$ sucrose water (Synergy Ultrapure Water Systems, Merck Millipore, France) suspension preheated to $55^{\circ} \mathrm{C}$. The round flask was then flushed with $\mathrm{N}_{2}$ gas before sealed with a rubber sealing, and put into a large beaker with water preheated to $55^{\circ} \mathrm{C}$ in a Styrofoam box, sealed and left overnight at the same temperature. The so-produced GUVs were extracted with a pipette and gently extruded 11 times by hand at $55^{\circ} \mathrm{C}$ through a $\varnothing=800 \mathrm{~nm}$ porous membrane. The vesicle size distribution was bimodal with a very strong peak around $r=140 \mathrm{~nm}$, and a barely visible peak around $r=250 \mathrm{~nm}$ according to a NTA analysis. To change the original distribution towards larger sized vesicles, four centrifugation steps were employed using a benchtop MIKRO 2 centrifuge 2R (Hettich, Germany). In each step, larger sized vesicles were pelleted, and the supernatant with smaller sized vesicles was discarded, while the pellet was resuspended in Milli-Q water. The resulting size distribution is shown in Fig. 5(b).

The vesicle sample with a monodisperse size distribution (Fig. 5(a)) was produced by rehydrating the lipid film made as described above in buffer $(100 \mathrm{mM} \mathrm{NaCl}, 10 \mathrm{mM}$
Tris (Sigma-Aldrich), pH 7.4) at room temperature and subsequently subjected to 10 cycles of freeze-thawing (liquid $\mathrm{N}_{2}$, water bath at $55^{\circ} \mathrm{C}$ ), and then extruded 31 times, by hand in a mini extruder (Avanti), through a $\varnothing=400 \mathrm{~nm}$ porous membrane (Whatman, UK). All vesicle solutions were then stored under $\mathrm{N}_{2}$-gas in sealed containers at $4{ }^{\circ} \mathrm{C}$ until use. The same buffer $(100 \mathrm{mM} \mathrm{NaCl}, 10 \mathrm{mM}$ Tris, $\mathrm{pH}$ 7.4) was used for both vesicle batches throughout the surface-immobilization and imaging experiments.

The ATTO-dye fluorescent phospholipids used in our work are labelled at the hydrophilic head group. After incorporation of these lipids into a lipid membrane, the fluorophore molecules are located at the water/lipid interface on both sides of the membrane and can be oriented in different directions, because the fluorophore-lipoid link is flexible. The monodisperse vesicles had on average $\sim 1000$ fluorophores per vesicle, while the vesicles with the sizes close to the first and second peak of the bimodal distribution had $\sim 4000$ and $\sim 13000$ fluorophores, respectively.

\section{Determination of vesicle sizes in solution}

Vesicle-size distributions in solution were measured using NTA with a NanoSight LM10 (NanoSight, United Kingdom), because this method is considered to be more accurate for measuring polydisperse distributions than, e.g., DLS. ${ }^{40,44,52}$ Data were typically obtained by multiple measurements with samples at a liposome concentration of $0.001 \mathrm{mg} / \mathrm{ml}$. In order to improve statistics for the more diluted bimodal samples, measurements were performed under a $2 \mu \mathrm{l} / \mathrm{min}$ flow provided by a syringe pump (NE-1000, New Era Pump Systems, Inc., USA). The corresponding results were then pooled together to increase the collected number of completed tracks to around $10^{4}$ and $3 \times 10^{4}$ for the monodisperse and bimodal samples, respectively.

\section{Surface modification}

Glass substrates (microscope coverslip \#1, Menzel Gläser, Braunschweig, Germany) were washed in Liqui-nox (Sigma-Aldrich) at $\simeq 95^{\circ} \mathrm{C}$ for about $1 \mathrm{~h}$. A glass substrate was then rinsed with a $10 \mathrm{mM}$ sodium dodecyl sulfate (SDS) solution before being thoroughly rinsed with milliQ-water (Millipore, France).

Vesicles were immobilized using biotin-streptavidin chemistry, as described previously. ${ }^{53}$ In brief, a $10 \mu \mathrm{g} / \mathrm{ml}$ solution of PLL-g-PEG/PLL-g-PEG-Biotin (PLL(20)-g[3.5]PEG(2), PLL(20)-g[3.5]-PEG(2)/PEG(3.4)-Biotin $\quad\left(1: 10^{5}\right.$ molar ratio, yielding approximately 2 biotins per $\mu \mathrm{m}^{2}$; Surface Solutions, Switzerland) in buffer was first introduced to the flow cell using a gravity driven flow, after which it was incubated for $\sim 45 \mathrm{~min}$. After subsequent rinsing, a solution of NeutrAvedin (10 mg/ml; Sigma-Aldrich, Germany) was injected and incubated for $30 \mathrm{~min}$. The monodisperse vesicle suspension was diluted to $0.001 \mathrm{mg} / \mathrm{ml}$ (lipid) concentration in buffer and flowed over the surface until a high enough vesicle coverage was reached. For the bimodal case, the concentration of the vesicle suspension was uncertain due to multiple centrifugation steps, but $200 \mu \mathrm{l}$ of the vesicle suspension remaining after the final centrifugation 
step was diluted to $1 \mathrm{ml}$ in buffer and flowed over the surface until a satisfactory surface coverage was reached. Finally, the measurement cell was rinsed with buffer before about 20 images were acquired in TIRFM mode to yield a total of about $10^{4}$-recorded vesicles for both the monodisperse and the bimodal sample. A low biotin surface coverage (PLL-g-PEGBiotin:PLL-g-PEG ratio of $1: 10^{5}$ ) was chosen to ensure that the separation of individual lipid vesicles was larger than the diffraction limit, a requirement for TIRFM-based single-vesicle identification. Each vesicle in the monodisperse vesicle sample had on average about 3 available biotin moieties on the outer membrane, while the bimodal vesicles, due to their larger size, had about 10 biotins moieties available for the peak at $r=140 \mathrm{~nm}$ and $\sim 35$ for the peak at $r=250 \mathrm{~nm}$. The low biotin coverages on the TIRFM substrate and the vesicles were chosen to reduce the number of anchoring points and potential vesicle deformation. $^{54}$

Taking the sizes of the counterparts of the biotinstreptavidin tether into account, ${ }^{55}$ the tether length is estimated to be $15-17 \mathrm{~nm}$. This length is rather large, and accordingly, the effect of the glass/water interface on the dye emission is nearly negligible even if dyes are located at the lower vesicle part.

\section{Microscopy imaging}

After cleaning and drying with $\mathrm{N}_{2}$-gas as described above, the substrate was put in a custom made flow cell, operated by a gravity driven flow. The flow cell was then mounted in an inverted fluorescence microscope (Nikon Eclipse Ti-E, Nikon Corporation, Japan) equipped with an EM-CCD camera (Andor iXon-DU897, Andor Technology, Northern Ireland), a mercury lamp connected with a fiberoptic cable (Intensilight C-HGFIE, Nikon Corporation), and a $60 \times$ oil immersion TIRFM-objective. With this equipment, the vesicle identification and intensity determination included a few steps. First, a coarse estimate for the background count rate was manually derived by measuring the count rate in regions that were clearly free from vesicles. As the single pixel background count rate scatters less than $5 \%$ around its average value (as indicated by measurements without any fluorescence excitation), a threshold of $108 \%$ of the background count rate reliably distinguishes "background pixels" (<108\%) from "vesicle pixels" $(>108 \%)$. A refined, average background count rate was determined by taking the median count rate of all background pixels. Vesicles were subsequently identified in the resulting image if at least all four adjacent pixels were above the threshold value. The corresponding pixel intensity per vesicle was corrected from the background by subtracting the refined, average background with the vesicle intensity selected as the sum of these values. The robustness of the selection was verified by making sure that a slight variation in the threshold value did not significantly influence the intensity distribution in the lower end of the histogram.

\section{Correlation of size distributions obtained from TIRFM and NTA}

For all the imaged vesicles, their integrated intensities were converted to corresponding vesicle radii using the relation between the measured intensity and size given by Eqs. (3) and (20) with $\delta=100 \mathrm{~nm}$ determined by using the conventional expression (see Appendix A; the experiments reported in Ref. 56 indicate that the accuracy of this expression is about $\pm 10 \%)$. This was done for different values (between 0.01 and 1 , with increments of 0.001 ) of the $A J_{0}$ prefactor in Eqs. (3) and (20). Size distributions were then created by making histograms of the calculated radii with a binning width of $0.5 \mathrm{~nm}$ (corresponding to the binning used for the NTA data), with all size distributions normalized to the magnitude of the strongest peak (this normalization is slightly preferable compared to the conventional normalization with respect to the total number of vesicles, because it facilitates the comparison of the distributions near the main peak). The value of the $A J_{0}$ prefactor that yielded the best fit to the position of the main peak in the NTA size distribution was then determined in each case. The curve fits were made using smoothing splines to determine the peak position by calculating its centroid for each fit. The smoothing parameter was set by eye to best match the smoothing level seen in the NTA data. The peak positions used for correlation were determined by calculating centroids of $20 \%$ of the top of the main peaks.

${ }^{1}$ O. G. Berg and M. K. Jain, Interfacial Enzyme Kinetics (Wiley, London, 2002); E. A. Dennis, J. Cao, Y.-H. Hsu, V. Magrioti, and G. Kokotos, Chem. Rev. 111, 6130 (2011).

${ }^{2}$ S. Urdy, Biol. Rev. 87, 786 (2012); M. Takeichi, Nat. Rev. Mol. Cell Biol. 15, 397 (2014).

${ }^{3}$ R. Jahn and D. Fasshauer, Nature 490, 201 (2012); S. Y. Shvartsman and R. E. Baker, WIREs Dev. Biol. 1, 715 (2012).

${ }^{4}$ J. Mercer, M. Schelhaas, and A. Helenius, Annu. Rev. Biochem. 79, 803 (2010); J. C. Tilton and R. W. Doms, Antiviral Res. 85, 91 (2010).

${ }^{5}$ D. Lingwood and K. Simons, Science 327, 46 (2010); O. G. Mouritsen, Phys. Chem. Chem. Phys. 13, 19195 (2011).

${ }^{6}$ B. Antonny, Annu. Rev. Biochem. 80, 101 (2011); T. Baumgart, B. R. Capraro, C. Zhu, and S. L. Das, Annu. Rev. Phys. Chem. 62, 483 (2011); J. A. Jackman, G. H. Zan, V. P. Zhdanov, and N.-J. Cho, J. Phys. Chem. B 117, 16117 (2013).

${ }^{7}$ R. Lipowsky, Adv. Colloid Interface Sci. 208, 14 (2014); V. P. Zhdanov and F. Höök, Biophys. Chem. 170, 17 (2012).

${ }^{8}$ G. J. Hardy, R. Nayak, and S. Zauscher, Curr. Opin. Colloid Interface Sci. 18, 448 (2013); T. G. Pomorski, T. Nylander, and M. Cardenas, Adv. Colloid Interface Sci. 205, 207 (2014).

${ }^{9}$ J. A. Jackman and N.-J. Cho, Biointerphases 7, 18 (2012).

${ }^{10}$ S. R. Tabaei, J.-H. Choi, G. H. Zan, V. P. Zhdanov, and N.-J. Cho, Langmuir 30, 10363 (2014); S. R. Tabaei, J. A. Jackman, S.-O. Kim, V. P. Zhdanov, and N.-J. Cho, Langmuir 31, 3125 (2015).

${ }^{11}$ B. O. Liedberg, C. Nylander, and I. Lundström, Sens. Actuators, A 4, 299 (1983); I. Abdulhalim, M. Zourob, and A. Lakhtakia, Electromagnetics 28, 214 (2008).

${ }^{12}$ E. M. Larsson, M. E. Edvardsson, C. Langhammer, I. Zorić, and B. Kasemo, Rev. Sci. Instrum. 80, 125105 (2009).

${ }^{13}$ D. L. M. Rupert, C. L. M. Eldh, S. Block, V. P. Zhdanov, J. O. Lotvall, M. Bally, and F. Höök, Anal. Chem. 86, 5929 (2014); E. Oh, J. A. Jackman, S. Yorulmaz, V. P. Zhdanov, H. Lee, and N.-J. Cho, Langmuir 31, 771 (2015).

${ }^{14}$ N. S. Hatzakis, V. K. Bhatia, J. Larsen, K. L. Madsen, P.-Y. Bolinger, A. H. Kunding, J. Castillo, U. Gether, P. Hedegar, and D. Stamou, Nat. Chem. Biol. 5, 835 (2009).

${ }^{15}$ A. H. Kunding, M. W. Mortensen, S. M. Christensen, and D. Stamou, Biophys. J. 95, 1176 (2008). 
${ }^{16}$ V. K. Bhatia, K. L. Madsen, P.-Y. Bolinger, A. Kunding, P. Hedegard, U. Gether, and D. Stamou, EMBO J. 28, 3303 (2009); M. B. Jensen, V. K. Bhatia, C. C. Jao, J. E. Rasmussen, S. L. Pedersen, K. J. Jensen, R. Langen, and D. Stamou, J. Biol. Chem. 286, 42603 (2011); A. Tonnesen, S. M. Christensen, V. Tkach, and D. Stamou, Biophys. J. 106, 201 (2014).

${ }^{17}$ S. R. Tabaei, M. Rabe, V. P. Zhdanov, N.-J. Cho, and F. Höök, Nano Lett. 12, 5719 (2012).

${ }^{18}$ S. Fujii, T. Matsuura, and T. Yomo, ACS Chem. Biol. 10, 1694 (2015).

${ }^{19}$ N. S. Hatzakis, L. Wei, S. K. Jorgensen, A. H. Kunding, P.-Y. Bolinger, N. Ehrlich, I. Makarov, M. Skjot, A. Svendsen, P. Hedegard, and D. Stamou, J. Am. Chem. Soc. 134, 9296 (2012).

${ }^{20}$ S. R. Tabaei, M. Rabe, H. Zetterberg, V. P. Zhdanov, and F. Höök, J. Am. Chem. Soc. 135, 14151 (2013).

${ }^{21}$ M. Rabe, S. R. Tabaei, H. Zetterberg, V. P. Zhdanov, and F. Höök, Angew. Chem. Int. Ed. 54, 1022 (2015).

${ }^{22}$ B. Lohse, P.-Y. Bolinger, and D. Stamou, J. Am. Chem. Soc. 130, 14372 (2008); N. Ehrlich, A. L. Christensen, and D. Stamou, Anal. Chem. 83, 8169 (2011).

${ }^{23}$ S. M. Christensen, M. W. Mortensen, and D. G. Stamou, Biophys. J. 100, 957 (2011); A. H. Kunding, M. W. Mortensen, S. M. Christensen, V. K. Bhatia, I. Makarov, R. Metzler, and D. Stamou, Biophys. J. 101, 2693 (2011).

${ }^{24}$ M. Bally, A. Gunnarsson, L. Svensson, G. Larson, V. P. Zhdanov, and F. Höök, Phys. Rev. Lett. 107, 188103 (2011); A. Gunnarsson, A. Snijder, J. Hicks, J. Gunnarsson, F. Höök, and S. Geschwindner, Anal. Chem. 87, $4100(2015)$

${ }^{25}$ D. Axelrod, T. P. Burghardt, and N. L. Thompson, Annu. Rev. Biophys. Bioeng. 13, 247 (1984).

${ }^{26}$ T. Wazawa and M. Ueda, Adv. Biochem. Eng. Biotechnol. 95, 77 (2005); H. Schneckenburger, Curr. Opin. Biotechnol. 16, 13 (2005); N. L. Thompson, X. Wang, and P. Navaratnarajah, J. Struct. Biol. 168, 95 (2009).

${ }^{27}$ S. M. Nie and R. N. Zare, Annu. Rev. Biophys. Biomol. Struct. 26, 567 (1997); X. Michalet, S. Weiss, and M. Jager, Chem. Rev. 106, 1785 (2006).

${ }^{28}$ D. Zheng, L. Kaldaras, and H. P. Lu, Rev. Sci. Instrum. 83, 013110 (2012).

${ }^{29}$ E. Boukobza, A. Sonnenfeld, and G. Haran, J. Phys. Chem. B 105, 12165 (2001); B. Okumus, T. J. Wilson, D. M. J. Lilley, and T. Ha, Biophys. J. 87, 2798 (2004).

${ }^{30}$ V. Filipe, A. Hawe, and W. Jiskoot, Pharm. Res. 27, 796 (2010).

${ }^{31}$ J. Pencer and F. R. Hallett, Langmuir 19, 7488 (2003).

${ }^{32}$ J. Larsen, N. S. Hatzakis, and D. Stamou, J. Am. Chem. Soc. 133, 10685 (2011).

${ }^{33}$ J. Y. Lee, S.-K. Kim, and S.-C. Hong, J. Korean Phys. Soc. 50, 1340 (2007).

${ }^{34}$ J. N. Greeson and R. M. Raphael, J. Biomed. Opt. 12, 021002 (2007).

${ }^{35}$ C. Gell, M. Berndt, J. Enderlein, and S. Diez, J. Microsc. 234, 38 (2009).

${ }^{36}$ J. Oreopoulos and C. M. Yip, Biophys. J. 96, 1970 (2009); P. Ferrand, P. Gasecka, A. Kress, X. Wang, F.-Z. Bioud, J. Duboisset, and S. Brasselet, Biophys. J. 106, 2330 (2014); Š. Timr, A. Bondar, L. Cwiklik, M. Štefl, M. Hof, M. Vazdar, J. Lazar, and P. Jungwirth, J. Phys. Chem. B 118, 855 (2014)

${ }^{37}$ H. S. Carslaw and J. C. Jaeger, Conduction of Heat in Solids (Oxford University Press, Oxford, 1959); V. P. Zhdanov and B. Kasemo, Proteins: Struct., Funct., Genet. 30, 177 (1998).

${ }^{38}$ V. G. Levich, Physicochemical Hydrodynamics (Prentice-Hall, New Jersey, 1962); V. P. Zhdanov, C. A. Keller, K. Glasmästar, and B. Kasemo, J. Chem. Phys. 112, 900 (2000).

${ }^{39}$ J. A. Jackman, V. P. Zhdanov, and N.-J. Cho, Langmuir 30, 9494 (2014).

${ }^{40}$ C. Lohr, A. H. Kunding, V. K. Bhatia, and D. Stamou, Methods Enzymol. 465, 143 (2009).

${ }^{41}$ S. R. Tabaeiab and N. J. Cho, Chem. Commun. 51, 10272 (2015).

${ }^{42}$ F. Olson, C. A. Hunt, F. C. Szoka, W. J. Vail, and D. Papahadjopoulos, Biochim. Biophys. Acta, Biomembr. 557, 9 (1979).

${ }^{43}$ M. J. Hope, M. B. Bally, G. Webb, and P. R. Cullis, Biochim. Biophys. Acta, Biomembr. 812, 55 (1985); M. Traikia, D. E. Warschawski, M. Recouvreur, J. Cartaud, and P. F. Devaux, Eur. Biophys. J. 29, 184 (2000);
J. A. Jackman, Z. Zhao, V. P. Zhdanov, C. W. Frank, and N.-J. Cho, Langmuir 30, 2152 (2014).

${ }^{44}$ R. A. Dragovic, C. Gardiner, A. S. Brooks, D. S. Tannetta, D. J. P. Ferguson, P. Hole, C. W. G. Redman, A. L. Harris, P. J. Dobson, P. Harrison, and I. L. Sargent, Nanomed. Nanotechnol. Biol. Med. 7, 780 (2011).

${ }^{45}$ S. M. Christensen, P.-Y. Bolinger, N. S. Hatzakis, M. W. Mortensen, and D. Stamou, Nat. Nanotechnol. 7, 51 (2012).

${ }^{46}$ D. Axelrod, Traffic 2, 764 (2001).

${ }^{47}$ C. Billaudeau, S. Mailfert, T. Trombik, N. Bertaux, V. Rouger, Y. Hamon, H.-T. He, and D. Marguet, Methods Enzymol. 519, 277 (2013); S. Rüttinger, Confocal Microscopy and Quantitative Single Molecule Techniques for Metrology in Molecular Medicine (Technischen Universität, Berlin, 2006).

${ }^{48}$ V. K. Bhatia, N. S. Hatzakis, and D. Stamou, Semin. Cell Dev. Biol. 21 381 (2010); S. M. Christensen and D. G. Stamou, Sensors 10, 11352 (2010); K. L. Madsen, V. K. Bhatia, U. Gether, and D. Stamou, FEBS Lett. 584, 1848 (2010); G. Drin and B. Antonny, FEBS Lett. 584, 1840 (2010); C. Chen, S. Zhu, T. Huang, S. Wang, and X. Yan, Anal. Methods 5, 2150 (2013); L. A. Bagatolli and D. Needham, Chem. Phys. Lipids 181, 99 (2014); A. Czogalla, M. Grzybek, W. Jones, and U. Coskun, Biochim. Biophys. Acta 1841, 1049 (2014).

${ }^{49}$ P. M. Bendix, M. S. Pedersen, and D. Stamou, Proc. Natl. Acad. Sci. U.S.A. 106, 12341 (2009); E.-Y. Lee, D.-Y. Choi, D.-K. Kim, J.-W. Kim, J. O. Park, S. Kim, S.-H. Kim, D. M. Desiderio, Y.-K. Ki1, K.-P. Kim, and Y. S. Gho, Proteomics 9, 5425 (2009); R. Luo, V. L. Ha, R. Hayashi, and P. A. Randazzo, Cell. Signalling 21, 1169 (2009); F. Delport, A. Deres, J.-I. Hotta, J. Pollet, B. Verbruggen, B. Sels, J. Hofkens, and J. Lammertyn, Langmuir 26(3), 1594 (2010); B. Liu, A. Mazouchi, and C. C. Gradinaru, J. Phys. Chem. B 114, 15191 (2010); P. M. Bendix and L. B. Oddershede, Nano Lett. 11, 5431 (2011); J. Bomholt, K. Moth-Poulsen, M. Harboe, A. O. Karlson, K. B. Qvist, T. Bjornholm, and D. G. Stamou, Langmuir 27, 866 (2011); B. W. Neuman, G. Kiss, A. H. Kunding, D. Bhella, M. F. Baksh, S. Connelly, B. Droese, J. P. Klaus, S. Makino, S. G. Sawicki, S. G. Siddell, D. G. Stamou, I. A. Wilson, P. Kuhn, and M. J. Buchmeier, J. Struct. Biol. 174, 11 (2011); I. Ammendrup-Johnsen, T. S. Thorsen, U. Gether, and K. L. Madsen, Biochemistry 51, 586 (2012); E. Elizondo, J. Larsen, N. S. Hatzakis, I. Cabrera, T. Bjornholm, J. Veciana, D. Stamou, and N. Ventosa, J. Am. Chem. Soc. 134, 1918 (2012); H. M. Piwonski, M. Goomanovsky, D. Bensimon, A. Horovitz, and G. Haran, Proc. Natl. Acad. Sci. U.S.A. 109, 8368 (2012); A. L. Christensen, C. Lohr, S. M. Christensen, and D. Stamou, Lab Chip 13, 3613 (2013); O. Friaa, M. Furukawa, A. Shamas-Din, B. Leber, D. W. Andrews, and C. Fradin, ChemPhysChem 14, 2476 (2013); Y.-S. Kim, E.-J. Choi, W.-H Lee, S.-J. Choi, T.-Y. Roh, J. Park, Y.-K. Jee, Z. Zhu, Y.-Y. Koh, Y. S. Gho, and Y.-K. Kim, Clin. Exp. Allergy 43, 443 (2013); J. C. Black, P. P. Cheney, T. Campbell, and M. K. Knowles, Soft Matter 10, 2016 (2014); S. Mathiasen, S. M. Christensen, J. J. Fung, S. G. F. Rasmussen, J. F. Fay, S. K. Jorgensen, S. Veshaguri, D. L. Farrens, M. Kiskowski, B. Kobilka, and D. Stamou, Nat. Methods 11, 931 (2014); R. Wyss, L. Grasso, C. Wolf, W. Grosse, D. Demurtas, and H. Vogel, Anal. Chem. 86, 7229 (2014).

${ }^{50}$ L. D. Landau and E. M. Lifshitz, Electrodynamics of Continuous Media (Pergamon, Oxford, 1984).

${ }^{51}$ K. Akashi, H. Miyata, H. Itoh, and K. Kinosita, Biophys. J. 71, 3242 (1996); A. Sapper, B. Reiss, A. Janshoff, and J. Wegener, Langmuir 22, 676 (2006).

${ }^{52}$ V. Sokolova, A.-K. Ludwig, S. Hornung, O. Rotan, P. A. Horn, M. Epple, and B. Giebel, Colloids Surf., B 87, 146 (2011).

${ }^{53}$ S. S. Perry, X. Yan, F. T. Limpoco, S. Lee, M. Müller, and N. D. Spencer, ACS Appl. Mater. Interfaces 1, 1224 (2009).

${ }^{54}$ B. Pignataro, C. Steinem, H. J. Galla, H. Fuchs, and A. Janshoff, Biophys. J. 78, 487 (2000).

${ }^{55}$ D. Clerc and W. Lukosz, Sens. Actuators, B 11, 461 (1993); T. Drobek, N. D. Spencer, and M. Heuberger, Macromolecules 38, 5254 (2005); O. Garbuzenko, Y. Barenholz, and A. Priev, Chem. Phys. Lipids 135, 117 (2005); M. Heuberger, T. Drobek, and N. D. Spencer, Biophys. J. 88, 495 (2005); F. Xu, G. Zhen, M. Textor, and W. Knoll, Biointerphases 1, 73 (2006).

${ }^{56}$ A. L. Mattheyses, J. Biomed. Opt. 11, 014006 (2006). 